\title{
Temporal landscape of human gut RNA and DNA virome in SARS-CoV-2 infection and severity
}

Tao Zuo

Qin Liu

Fen Zhang

Yun Kit Yeoh

Yating Wan

Hui Zhan

Grace C. Y. Lui

Zigui Chen

Amy Y. L. Li

Chun Pan Cheung

Nan Chen

Wenqi Lv

Rita W. Y. Ng

Eugene Y. K. Tso

Kitty S. C. Fung

Veronica Chan

Lowell Ling

Gavin Joynt

David S.C. Hui

Francis K. L. Chan

Paul K. S. Chan

Siew Ng

\section{Video Byte}

Keywords: COVID-19, SARS-CoV-2, coronavirus, COVID, virome, RNA, DNA, Microbiome

Posted Date: October 14th, 2021

DOI: https://doi.org/10.21203/rs.3.rs-968277/v1

License: (9) This work is licensed under a Creative Commons Attribution 4.0 International License. Read Full License 


\section{Abstract}

COVID-19, caused by the enveloped RNA virus SARS-CoV-2, primarily affects the respiratory and gastrointestinal systems. Disease severity varies substantially across patients; the disease is mild or asymptomatic in some cases and causes respiratory failure or death in others. SARS-CoV-2 viral RNA has been detected in fecal samples, suggesting that the gastrointestinal tract may be a site of viral replication. To better understand the effect of SARS-CoV-2 on resident gut viruses (the "gut virome"), researchers examined blood samples and fecal specimens from 98 patients with COVID-19 and 78 healthy individuals. Using shotgun metagenomics, they found that patients with COVID-19 had decreased abundance of certain RNA viruses and DNA-based bacteriophages. In contrast, environment-derived eukaryotic DNA viruses were enriched in COVID-19 patients. The fecal virome expressed more genes associated with stress, inflammation, and virulence in patients with COVID-19, and the baseline abundance of 10 different virus species was inversely correlated with virus severity and with the patients' age. These results suggest that COVID-19 may affect the composition of the gut virome in association with host stress responses and hyper-inflammation, highlighting the importance of the human gut virome in the severity of and potential therapeutics for COVID-19. 